\title{
Optimization of SPR signals: Monitoring the physical structures and refractive indices of prisms
}

\author{
Wan Maisarah Mukhtar ${ }^{1, *}$, Razman Mohd Halim², Hazirah Hassan ${ }^{I}$ \\ ${ }^{1}$ Faculty of Science and Technology, Universiti Sains Islam Malaysia (USIM), Bandar Baru Nilai, 71800 Nilai, Negeri Sembilan, \\ Malaysia \\ ${ }^{2}$ National Metrology Institute of Malaysia, Lot PT 4803, Bandar Baru Salak Tinggi, 43900 Sepang, Selangor
}

\begin{abstract}
Surface plasmon resonance (SPR) can only be achieved if sufficient energy is provided at the boundary between metal and dielectric. An employment of prism as a light coupler by using Kretschmann configuration is one of the alternative for the production of adequate energy to be generated as surface plasmon polaritons (SPP). This work is carried out to investigate the effect of physical structure of the prism and its refractive index to the excitation of SPPs. A 50nm gold thin metal film with dielectric constant of $\varepsilon=-12.45 \mathrm{i}+1.3$ was deposited on the hypotenuse surface of the prisms. The physical structures of the prisms were varied such as triangular, conical, hemispherical and half cylindrical. These prisms were classified into two types of refractive indices (RI), namely $n=1.51$ (type BK7) and $n=1.77$ (type SF11). Based on SPR curve analyses, we discovered that strong SPR signals which consist of $82.98 \%$ photons were excited as SPPs can be obtained by using type-BK7 prism with physical structures of hemispherical or half cylindrical. From the view of selectivity ability as sensors, the usage of type-SF11 prisms (half cylindrical and hemispherical) able to enhance this impressive feature in which sharp SPR curves with small FWHM values were obtained. In conclusion, apart from properties of thin film materials, the physical structure of prisms and their RI values play crucial roles to obtain optimum SPR signal. High sensitivity SPR sensor can be established with the appointment of type-BK7 prisms (hemispherical or half cylindrical shape) as light couplers.
\end{abstract}

\section{Introduction}

An evolution of plasmonic technology is growth rapidly nowadays due to its ability in light manipulation which make it a suitable candidate in the development of optoelectronic device technology [1-5]. Plasmonic wave properties that react at the interface of the optical component which used nanophotonic structures made it as one of the favourable sensing techniques, have been widely used as surface plasmon resonance (SPR) sensor [6-8].

To enhance the sensitivity of SPR sensor, researchers introduced nanomaterials such as chitosan $[9,10]$, zinc oxide [11,12], gold nanoparticle [13,14], graphene $[15,16]$ and graphene oxide $[17,18]$. Obviously, the employment of those materials results positive responds where the sensitivity of sensors is successfully increased. The synthesis processes of these materials involve complicated procedure and costly materials such as seed-mediated growth technique, plasma enhance chemical vapor deposition and Hummer's method [14, 17,18].

Apart of hiring nanomaterials, an optimization of SPR setup able to generate maximum excitation of surface plasmon polaritons. Basic SPR setup consists of laser, lens, polarizer, prism, thin film and photodetector
[19]. Each component contributes its own significant role. Laser light must be oriented in p-polarized mode so that the electrons' interaction between metal and dielectric will create SPPs oscillations due to the applied electromagnetic field [20]. Note that suitable types of metal thin film with appropriate thicknesses, usually $50 \mathrm{~nm}$ also need to be considered [21].

In free space optics, light energy is collected from open beams and surfaces and sent on to the photodetector. SPR involved a free space optics application in which an appropriate light coupling method need to be employ so that sufficient energy for the excitation of surface plasmon polaritons can be produced. One of the common alternatives is the usage of prism as light coupler. The prism is designed to couple a substantial fraction of the power contained in a laser beam into a metal thin film [22]. Ignoring the presence of prism will result unsuccessful SPR phenomena. To date, there are two types of prism coupling configuration for SPR application which are familiarly used; namely Kretschmann configuration [23] and Otto configuration [24]. Kretschmann configuration is preferable due to its simplicity.

In this work, the important control parameters that influence the strength of SPR signal are studied for the improvement of SPP excitations. We investigate the

\footnotetext{
* Corresponding author: wmaisarah@usim.edu.my
} 
effect of physical structure and refractive index (RI) of prisms on SPR. We believe that the shape of prism and its RI value play significant roles to enhance the SPR signal, in which leads to the development of a low cost and high sensitivity optical sensor.

\section{Theory}

Surface plasmon is the localized charge density oscillations that propagate along the boundary between a thin metal film and a dielectric medium. The excitation of surface plasmon polaritons (SPP) can be established as the conservation of both energy and momentum occurs. To achieve this, the relation between energy (in terms of the angular frequency $\omega$ ) and the momentum (in terms of the wavevector in the propagation direction $k_{x}$ ) need to be analysed. An important characteristics of surface plasmons is that for a given energy $\hbar \omega$, the wavevector $k_{x}$ is always larger than the wavevector of light in free space as illustrated in Figure 1. The light line $\omega / c$ is plotted as dashed lines. The strong coupling between light and surface charges increased the momentum of SPP; where the electrons along the metal surface has been dragged by the light field. This phenomenon describes the condition where SPP on a plane interface cannot be excited by light of any frequency that propagates in free space. Excitation of SPP by light is only possible if a wavevector component of the exciting light can be increased over its free-space value. One of the alternative solution is by created the evanescent field at the interface between a medium with refractive index $>1$, where the light will be tilted by a factor of $\mathrm{n}$ since $\omega=c k / n$. This situation is shown in Figure 1, which portrays the SPP dispersion with the free-space light line and the tilted light line in glass with various refractive indices such as $n_{1}, n_{2}$ and $n_{3}$ where $n_{1}$ $<n_{2}<n_{3}$. As the light lines cross the SPP curve, the energy and momentum are conserved which resulting the occurrence of SPR phenomenon [22].

The relationship of the surface plasmon wave vector $\left(k_{s p p}\right)$ between the metal and dielectric medium is given as

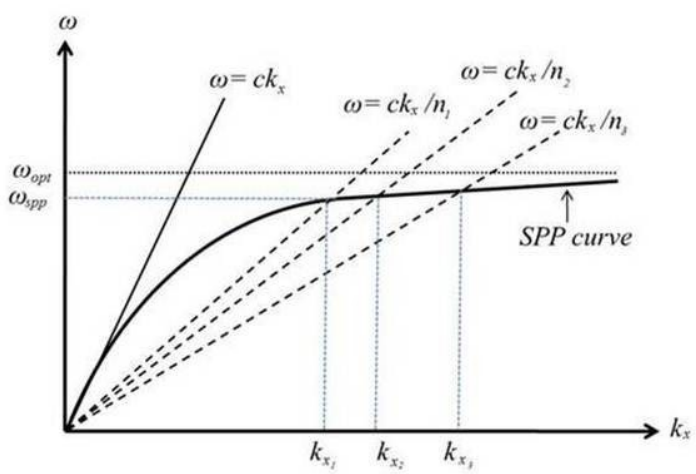

Fig. 1. SPP dispersion with the free-space light line and the tilted light line in glass with various refractive indices such as $n_{1}, n_{2}$ and $n_{3}$ where $n_{1}<n_{2}<n_{3}$.

$$
k_{\text {spp }}=k_{o} \sqrt{\frac{\varepsilon_{\text {metal }} \varepsilon_{\text {dielectric }}}{\varepsilon_{\text {metal }}+\varepsilon_{\text {dielectric }}}}
$$

where $k_{o}$ is the free space wave vector of the optical wave, $\varepsilon_{\text {metal }}$ the dielectric functions of metal and $\varepsilon_{\text {dielectric }}$ is the dielectric functions of metal and the dielectric medium [25]. During SPR, the energy of the incident light can be strongly coupled from photons onto the surface plasmon wave which decays via heat dissipation along the propagation path. The relationship between $k_{x}$ and angle of incident light can be expressed as follows [26]:

$$
k_{x}=k_{o} n_{\text {glass }} \sin \theta_{\text {inc }}
$$

where $n_{\text {glass }}$ is the refractive index of the glass prism and $\theta_{\text {inc }}$ is the angle of incidence [17]. The conditions for the surface plasmon excitation at the interface between the metal and dielectric layers in a glass-metal-dielectric are given by

$$
k_{x}=k_{s p p}
$$

Resonance is achieved by matching the projection of the wave vector of the incident light in the direction of the interface and wave vector of the surface plasmon oscillations as expressed in Eq. (3). Using a prism with dielectric constant value larger than 1 with the incident angle is larger than the critical angle, value of $k_{x}$ can be determined. As the incident light satisfies the resonance condition, a strong absorption dip in the reflectance can be observed.

SPP wavelength, $\lambda_{S P P}$ is a wavelength during surface plasmon resonance phenomena which represents by a sharp dip curve. The SPP wavelength can be calculated from the derivation of Eq. 1, which expressed in Eq. 4 and 5 as follow [26]:

$$
\begin{aligned}
& \frac{2 \pi}{\lambda_{\text {spp }}}=\frac{2 \pi}{\lambda_{0}} \sqrt{\frac{\varepsilon_{\text {metal }} \varepsilon_{\text {dielectric }}}{\varepsilon_{\text {metal }}+\varepsilon_{\text {dielectric }}}} \\
& \lambda_{\text {spp }}=\lambda_{0} \sqrt{\frac{\varepsilon_{\text {metal }}+\varepsilon_{\text {dielectric }}}{\varepsilon_{\text {metal }} \varepsilon_{\text {dielectric }}}}
\end{aligned}
$$

\section{Methodology}

A $50 \mathrm{~nm}$ gold thin film with dielectric constant of $\varepsilon=-12.45 \mathrm{i}+1.3$ was deposited on the hypotenuse surface of the prisms. Two types of commercialized prisms were used, namely a BK-7 prism with refractive index of 1.51 and SF11 prism with refractive index of 1.77. A He-Ne laser source with $632.8 \mathrm{~nm}$ excitation wavelength was strike on the prism. To observe the role of prisms' shape as light coupler; various designs of prism were employed namely triangular, conical, hemispherical and half cylindrical. Fig. 2 shows an illustration of SPR setup, where a half cylindrical prism was employed as light 
coupler. The resonant excitation of surface plasmons was characterized by a dip in the reflectance curves which refers to the energy of the incident light that is partially transferred to plasmons [22]. In this work, the characterization of SPR characteristics was conducted by calculating the depth of SPR curves. A good SPR sensor with high sensitivity can be evaluated by studying the SPR curve depth. The deeper the depth of SPR curve, the more the SPPs were excited [2,6,14]. A full-width-halfmaximum (FWHM) analysis is carried out to investigate the selectivity capability of SPR sensor. Sharper SPR dip will produce smaller value of FWHM, which leads to the excellent selectivity capability due to the improvement of sensor resolution [27].

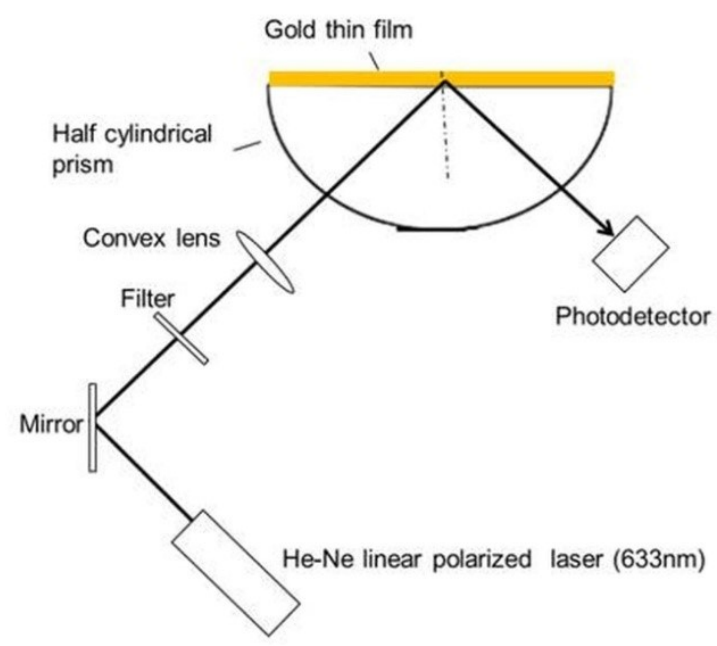

Fig. 2. Illustration of SPR setup using half cylindrical prism as light coupler.

\section{Results and discussions}

Fig.3 depicts the SPR curves for various structures of SF11 prisms consist of conical, hemispherical, half cylindrical and triangular structures; with refractive index of 1.77 (type SF11). Strong SPR signals were obtained for all prisms where two groups of SPR curves were obviously seen. The cone and triangular prisms displayed same SPR curves criteria, as well as hemisphere and half cylindrical prisms. To facilitate the analyses, these groups were classified as follows: Group 1 consists of conical and triangular prisms; Group 2 comprises of hemispherical and half cylindrical prisms. These groups exhibited significant differences of SPR curves. For Group 1, the values of minimum reflectance, $R$ and SPR curve depth, $\Delta \mathrm{R}$ were obtained as $R=0.0363$ and $\Delta R=0.7741$ respectively where the resonant angle is obtained at $\theta_{R}=28.6935^{\circ}$ and $\mathrm{FWHM}=1.44$. Meanwhile for Group 2, the values obtained were $R=0.0233$, $\Delta R=0.7684, \theta_{R}=35.9900^{\circ}$ and $\mathrm{FWHM}=0.83$.

The usage of SF11 prisms with conical and triangular shapes able to generate strong SPR signal where $77.41 \%$ of input beam was excited as SPP. Group
1 capable to produce stronger SPR signal which is $1.201 \%$ better than Group 2. The capability of SPR sensor is also determined by its selectivity [29]. We found that Group 2 (hemisphere and half cylindrical prisms) exhibited smaller values of FWHM in which indicated an excellent selectivity capability as compare to Group 1. The contradiction between these parameters demonstrates that the performance of SPR sensor can be categorized into two parts, namely sensitivity (due to the strong excitation of SPPs) and selectivity (due to the sharpness of the SPR curves).

Subtle differences were obtained as SF11 prisms $(\mathrm{n}=1.77)$ were replaced with BK7 prisms $(\mathrm{n}=1.51)$ where the resonant angles were red-shifted about $35 \%$ appeared as depicted in Fig.4. Obviously, the hemispherical and half cylindrical prisms exhibited identical SPR properties as well as triangular and cone. By applying the same approach of prism grouping (classification of Group 1 and Group 2 based on prism's structure), the performance of BK7 prisms as light coupler were investigated. For Group 1, the value of minimum reflectance, $R$ and SPR curve depth, $\Delta R$ were obtained as $R=0.0404$ and $\Delta R=0.8212$ respectively with the resonant angle at $\theta_{R}=43.1658^{\circ}$ and FWHM $=1.72$. Meanwhile for Group 2, the values obtained were $R=0.0310, \Delta R=0.0 .8298, \theta_{R}=43.7688^{\circ}$ and $\mathrm{FWHM}=1.16$.

Contrary with SF11 prisms, strong SPR signals were obtained as type BK-7 prisms consists of half cylindrical and hemispherical structures were employed where about $82.98 \%$ of photons were generated as SPPs. Group 2 results better SPR signal which is $1.036 \%$ of reflected light than Group 1. FWHM analyses explained that excellent selectivity of SPR sensing properties can be obtained by using hemispherical and half cylindrical prisms in which the values of FWHM were smaller than conical and triangular prisms. By employing hemispherical or half cylindrical type-BK 7 prisms, it can be concluded that high sensitivity and excellent selectivity SPR sensor can be developed. Table 1 lists SPR parameters which were obtained using two types' prisms with different RI.

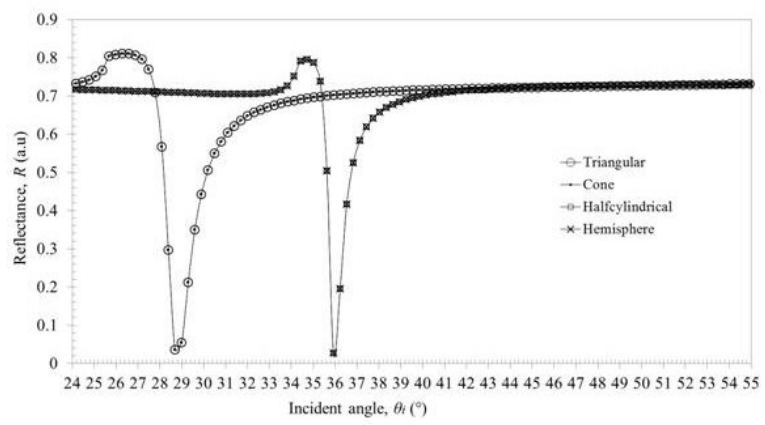

Fig. 3. SPR curves for various structures of SF11 prisms $(\mathrm{n}=1.77)$ consist of conical, hemispherical, half cylindrical and triangular. 


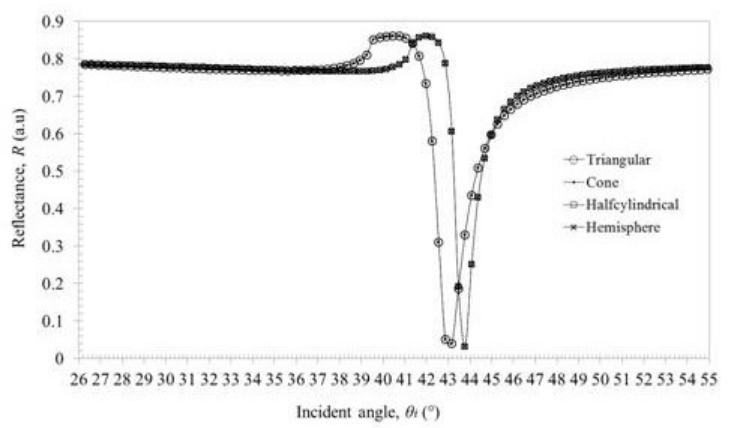

Fig.4. SPR curves for various structures of BK7 prisms $(\mathrm{n}=1.51)$ consist of conical, hemispherical, half cylindrical and triangular

Table 1. Important parameters of SPR signals

\begin{tabular}{clrrrrr}
\hline \multirow{2}{*}{ Type } & \multicolumn{1}{c}{ Shape } & $\begin{array}{c}\text { Minimum } \\
\text { reflectance, } R \text { (a.u) }\end{array}$ & $\begin{array}{c}\text { Resonant angle, } \\
\theta\left({ }^{\circ}\right)\end{array}$ & $\begin{array}{c}\text { SPR curve } \\
\text { depth, } \Delta R \text { (a.u) }\end{array}$ & FWHM, $\Delta \theta\left(^{\circ}\right)$ \\
\hline \hline \multirow{2}{*}{$\begin{array}{c}\text { SF11 Prism } \\
(\mathrm{n}=1.77)\end{array}$} & Triangular & Cone & 0.0363 & 28.6935 & 0.7741 & 1.6200 \\
& Halfcylindrical & 0.0363 & 28.6935 & 0.7741 & 1.6200 \\
& Hemisphere & 0.0261 & 35.9900 & 0.7684 & 0.8800 \\
\hline \multirow{2}{*}{$\begin{array}{c}\text { BK7 Prism } \\
(\mathrm{n}=1.51)\end{array}$} & Triangular & 0.0261 & 35.9900 & 0.7684 & 0.8800 \\
\cline { 2 - 6 } & Cone & 0.0404 & 43.1658 & 0.8212 & 1.7200 \\
& Halfylindrical & 0.0404 & 43.1658 & 0.8212 & 1.7200 \\
\hline
\end{tabular}

Fig.5 displays the properties of SPR as the RI of prisms were manipulated. Different SPR angles were substantially observed as different RI of prisms employed. The usage of Group 1 prisms with SF11 type results SPR angles at $28.6935^{\circ}$ (Fig. 5(a)). By replacing the prism with BK7-type, the SPR angles were shifted about $50.43 \%$ to $43.1658^{\circ}$. The employment of BK7 prisms able to produce high sensitivity SPR sensor due to their capability to excite more SPPs, which is $6.08 \%$ than SF11. The appointment of Group 2 prisms results the shifting of SPR angle about $21.61 \%$ from $35.9900^{\circ}$ to $43.7688^{\circ}$. This outcome proves that apart of
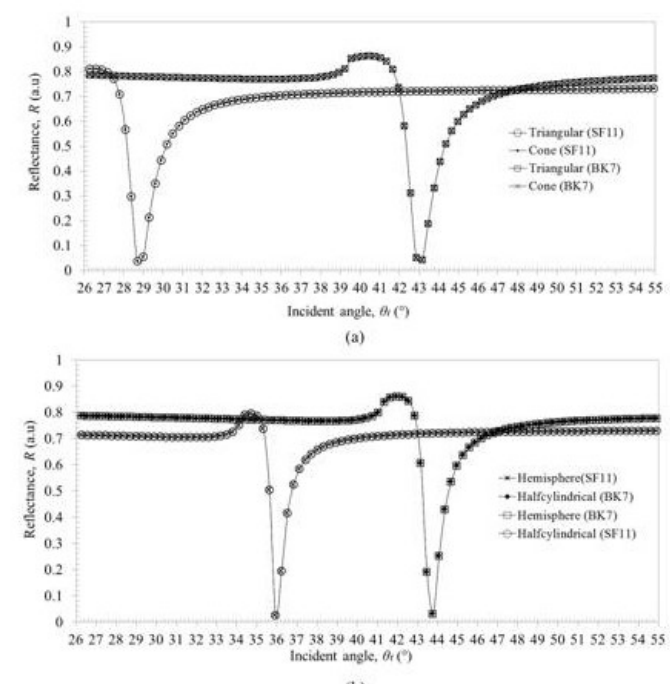

(b)

Fig. 5. SPR curves comparison between $\mathrm{SF} 11(\mathrm{n}=1.77)$ and BK7 $(\mathrm{n}=1.51)$ prisms with various physical structures (a)triangular and conical (b)hemispherical and half cylindrical.

thin film thicknesses, the physical structure and the value of RI of the prisms also play major roles for the generation of maximum amount of SPPs to optimize the SPR signal. The location of SPR angles are mainly influenced by RI's value of the light coupler. High sensitivity sensor due to strong SPR signal can be obtained by using Group 2 type-BK7 prism where $82.98 \%$ of photons were excited as SPPs (Fig. 6). From the view of excellent selectivity function as sensors [28], the usage of Group 2 type-SF11 prisms able to enhance this impressive feature in which sharp SPR curves with small FWHM values were obtained as summarized in Fig. 7.

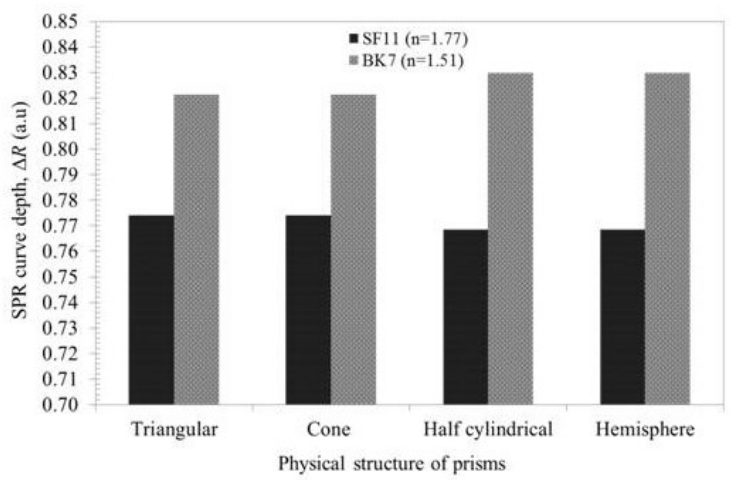

Fig. 6. Analysis of SPR curve depth versus various types of physical structure of prisms.

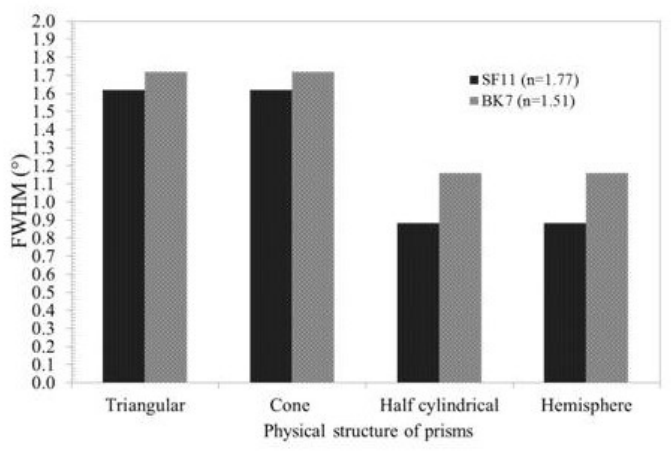

Fig. 7. FWHM analysis of SPR curves versus various types of physical structure of prisms

\section{Conclusions}

In this study, we successfully demonstrated the significant roles of physical structures of the prisms and their RI value which contribute to the remarkable SPR signals. The employment of half cylindrical or hemispherical prisms with low RI using BK7-type $(n=1.51)$ enhance the excitation of SPPs up to $8 \%$ in comparison with SF11-type $(n=1.77)$. This feature is one of the crucial criteria for the development of optical sensor. We believe that the output of this research will benefits to the rapid evolution of SPR optical sensing field.

The authors would like to acknowledge the support of the Universiti Sains Islam Malaysia and Malaysian Ministry of 
Education for funding this work under grant FRGS/2/2014/SG02/USIM/03/1. Knoll Group from Max Planck Institute for Polymer Research, German is also acknowledged for the Winspall 3.02 simulation software.

\section{References}

1. R. P. Dwivedi, H. S. Lee, J. H. Song, S. An, S, E. H. Lee, Opt Commun, 2841418 (2011)

2. W. M. Mukhtar, S. Shaari, A. A Ehsan, P. S. Menon, Opt Mat Exp, 4424 (2014).

3. V.E.Babicheva, A. V. Lavrinenko, Optics Communications, 2855500 (2012).

4. R. Thomas, Z. Ikonic, R. W. Kelsall, Photonic Nanostruct, 10183 (2012).

5. W. M. Mukhtar, S. Shaari, P. S. Menon, H. A. Razak, 2015 IEEE Regional Symposium, 2015, p.1.

6. W. M. Mukhtar, S. Shaari, P. S. Menon, Optoelectron Adv. Mat, 79 (2013).

7. J. Homola, S. S Yee, G. Gauglitz, Sensor Actuat BChem, 543 (1999).

8. A. G. Brolo, R. Gordon, B. Leathem, K. L. Kavanagh, Langmuir, 204813 (2004).

9. L. G. Zamfir, I. Geana, S. Bourigua, L. Rotariu, C. Bala, A. Errachid, N. Jaffrezic-Renault, Sensor Actuat B-Chem, 159178 (2011).

10. Y. W. Fen, W. M. M. Yunus, N. A. Yusof, Sensor Actuat B-Chem, 171287 (2012).

11. C. C. Chang, N. F. Chiu, D. S. Lin, Y. Chu-Su, Y. H. Liang, C. W. Lin, Anal Chem, 821207 (2010).

12. E. Sachet, M. D. Losego, J. Guske, S. Franzen, J. P. Maria, Appl Phys Lett, 10205111 (2013).

13. S. Ko, T. J. Park, H. S. Kim, J. H. Kim, Y. J. Cho Biosens Bioelectron, 242592 (2009).

14. W. M. Mukhtar, S. Shaari, P. S. Menon, Adv Sci Lett, 191412 (2013).

15. S. Szunerits, N. Maalouli, E. Wijaya, J. P. Vilcot, R. Boukherroub, Anal Bioanal Chem, 4051435 (2013).

16. L. Wang, C. Zhu, L. Han, L. Jin, M. Zhou, S. Dong, Chem Commun, 477794 (2011).

17. T. Wu, S. Liu, Y. Luo, W. Lu, L. Wang, X. Sun, Nanoscale, 32142 (2011).

18. S. K. Mishra, S. N. Tripathi, V. Choudhary, B. D. Gupta, Sensors Actuat B- Chem, 199190 (2014).

19. W. M. Mukhtar, P. S. Menon, S. Shaari, M. Z. Malek, A. M. Abdullah, J Phys Conf Ser, 431 012028 (2013).

20. W. B. Lin, N. Jaffrezic-Renault, A. Gagnaire, H. Gagnaire, Sensors Actuat A-Phys, 84198 (2000).

21. S. Zeng, K. V. Sreekanth, J. Shang, T. Yu, C. K. Chen, F. Yin, K. T. Yong, Adv Mat, 276163 (2015).

22. L. Novotny, B.Hecht, Principles of Nano-Optics. Cambridge University Press. United Kingdom. (2006).
23. S. Liu, M. Li, C. Gong, H. Gao, Laser Phys, 26 065006 (2016).

24. S. Maegawa, J. Yamaguchi, F. Itoigawa, T. Nakamura, Tribol Lett, 62 1(2016).

25. W. M. Mukhtar, S. Shaari, P. S. Menon, Adv Sci Lett, 1966 (2013).

26. M. S. Islam, A. Z. Kouzani, X. J. Dai, W. P. Michalski, Biomed Signal Proces, 6 147(2011).

27. A. Paliwal, A. Sharma, M. Tomar, V. Gupta, Sensors Actuat B-Chem, 216497 (2015).

28. N. Cennamo, G. D’Agostino, M. Pesavento, L. Zeni, Sensors Actuat B-Chem, 191529 (2014). 